\title{
RELIABILITY ELECTRICAL POWER SYSTEM OF HOSPITAL AS COLD STANDBY SYSTEM
}

\author{
NIEZAWODNOŚĆ SYTEMU \\ ELEKTROENERGETYCZNEGO SZPITALA JAKO \\ NIEZAWODNOŚĆ SYSTEMU Z REZERWĄ ZIMNĄ
}

\author{
Franciszek Grabski \\ Akademia Marynarki Wojennej \\ F.Grabski@amw.gdynia.pl
}

\begin{abstract}
The probabilistic model of a hospital electrical power system consisting of mains, an emergency power system and the automatic transfer switch with the generator starter are discussed in this paper. The reliability model is semi-Markov process describing two different units renewable cold standby system and switch. The embedded Semi-Markov processes concept is applied for description of the system evolution. Time to failure of the system is represented by a random variable denoting the first passage time of the process from the given state to the subset of states. The appropriate theorems of the Semi-Markov processes theory allow us to evaluate the reliability function and some reliability characteristics.
\end{abstract}

Keywords: semi-Markov model, renewable cold standby system, electrical power system

Streszczenie: $W$ pracy zostat przedstawiony model niezawodnościowy sytemu elektroenergetycznego szpitala złożony z podsystemu zasilania sieciowego, podsystemu zasilania awaryjnego oraz automatycznego przełacznika. Niezawodnościowym modelem funkcjonowania systemu jest proces semimarkowski. Model ten jest modyfikacja modelu niezawodności opisujacego funkcjonowanie sytemu $z$ rezerwa zimna złożonego $z$ dwóch różnych podsystemów i przełacznika. Do konstrukcji modelu zostat wykorzystany tak zwany włożony proces semi-markowski. Czas zdatności systemu jest reprezentowany przez czas pierwszego przejścia procesu do określonego podzbioru stanów.

Stowa kluczowe: model semi-markowski, system z rezerwa zimna, system elektroenergetyczny szpitala 
Reliability electrical power system of hospital as cold standby system

Niezawodność sytemu elektroenergetycznego szpitala jako niezawodność systemu...

\section{RELIABILITY ELECTRICAL POWER SYSTEM OF HOSPITAL AS COLD STANDBY SYSTEM}

\section{Introduction}

An electrical power system of a hospital consisting of the mains and the emergency power systems is discussed in the paper. Mains power of a hospital can be lost due to interruption of electrical lines during earthworks or in case of the overhead line of its destruction as a result of strong wind, heavy acing, malfunctions at a substation, planned blackouts or in extreme cases as a grid-wide failure. Loss of mains voltage may last from a few seconds to several hours or in extreme cases several days. The effects of such a situation may differ for different classes of devices. It leads to the automatic shutdown of equipment and there is a high risk of the equipment damage. It refers to life-saving equipment, anaesthesia apparatus, dialyzers in dialysis, or ultrasound devices. In the case of medical devices usually any loss of power causes distortion of its work as the device after restoring the power supply begins the process of self-testing and self-calibration is not performing at the time their basic functions. For other devices like elevators, refrigerators, ventilators a restoration of power is not a major problem. In hospitals most emergency power systems have been and are still based on generators. Electric generator supplies electricity selected electrical circuits and electric appliances in the hospital in an emergency (no power from the power grid). Through the hospital's power generator we understand set of devices consisting of an internal combustion or Diesel engine, generator and automatic detection system if there is no voltage in the power grid and the self actuating generator. A separate issue is the switching time. Usually, it ranges from a few to several seconds. The generator starts automatically. With regular generators, an automatic transfer switch is used to connect emergency power.

\section{Assumptions of model}

To evaluate the reliability characteristics of the hospital electrical power system a semi-Markov model is constructed. The model is modification of two different units cold standby system with switch [3], [5], [6]. In this case an operating unit $A$ denotes mains power system (subsystem), the stand-by unit $B$ means emergency power system (subsystem) and a switch is the automatic transfer switch with the generator starter. We should mention that two units renewable cold standby system was discussed in [1] and [7]. The time to failure of the mains power subsystems is an exponential distributed random variable $\zeta_{A}$. When the mains $A$ fails, the emergency power subsystem $B$ is immediately put in motion by the switch, (switching time is omitted). The failed system is repaired. A repair time of the basic power system $A$ is a random variable $\gamma_{A}$ having distribution given by the PDF $h_{A}(x)=\mu_{A}^{2} x e^{-\mu_{A} x}, x \geq 0$. At once after repairing the mains power subsystem $A$ is put in motion by the switch. 
A time to failure of the emergency power $\operatorname{system} B$ is a random variable $\zeta_{B}$, with an exponential PDF. If the emergency power subsystem $B$ fails during repair period of the mains power subsystem $A$, then it follows damage to of the whole electricity power system. The failure of the system takes place when the mains power subsystem fails and the emergency subsystem fails before repairing the basic subsystem $A$ or when the subsystem $A$ fails and the switch fails. Let $U$ be a random variable having a binary distribution

$$
b(k)=P(U=k)=a^{k}(1-a)^{1-k}, \quad k=0,1, \quad 0<a<1,
$$

where $U=0$ if a switch is "down" at the moment of the subsystem $A$ failure or renewal and $U=1$ otherwise.

A restoring time of the whole power system is the random variable $\gamma$. Moreover we assume that all random variables mentioned above are independent. A main goal of this paper is an evaluation a reliability function and a mean time to failure of the hospital electrical power system. To solve the problem, 4 state semi-Markov reliability model is constructed. The parameters of the model were estimated on the basis of few data and the opinion of the technical staff at one of the hospital in Poland. Therefore values of these parameters should be treated as approximate ones. Moreover we assume that all random variables mentioned above are independent.

\section{Model construction}

To describe the reliability evolution of the system, we have to define the states and the renewal kernel. We introduce the following states:

0 - failure of the whole system due to a failure of a switch,

1 - failure of the whole system due to the failure of the subsystem $B$ during repair period of the subsystem $\mathrm{A}$,

2 - failure of the mains power subsystem $A$, the emergency power subsystem $B$ is working,

3 - both the mains power subsystem and emergency subsystem are "up" and system A is working.

We assume that 3 is the initial state. We construct a random process in a following way. Let $0=\tau_{0}^{*}, \tau_{1}^{*}, \tau_{2}^{*}, \ldots$ denote the moments of the state changes, and $\{Y(t): t \geq 0\}$ be a random process with the state space $S=\{0,1,2,3\}$, with the right-hand continuous trajectories keeping constant values on half-intervals $\left[\tau_{n}^{*}, \tau_{n+1}^{*}\right), n=0,1, \ldots$. This process is not semi-Markov, because a momoryless property is not satisfied for all instants of the state changes of it.

Let us construct a new process in the following way. Let $0=\tau_{0}$ and $\tau_{1}, \tau_{2}, \ldots$ denote instants of the power subsystems failures or instants of the subsystem or whole system repair. Let $\{X(t) ; t \geq 0\}$ be a stochastic process, defined by the rule

$$
X(0)=3, \quad X(t)=Y\left(\tau_{n}\right) \quad \text { dla } \quad t \in\left[\tau_{n}, \tau_{n+1}\right)
$$


Reliability electrical power system of hospital as cold standby system

Niezawodność sytemu elektroenergetycznego szpitala jako niezawodność systemu...

This process is the semi-Markov process and it is called an embedded semiMarkov process in the stochastic process $\{Y(t): t \geq 0\}$.

To determine a semi-Markov process as a model we have to define its initial distribution and all elements of its kernel [5], [6], [7]. Recall that the semi-Markov kernel is the matrix of transition probabilities of the Markov renewal process [6]

$$
\begin{gathered}
Q(t)=\left[Q_{i j}(t): i, j \in S\right], \\
Q_{i j}(t)=P\left(\tau_{n+1}-\tau_{n} \leq t, X\left(\tau_{n+1}\right)=j \mid X\left(\tau_{n}\right)=i\right), \quad t \geq 0 .
\end{gathered}
$$

The sequence $\left\{X\left(\tau_{n}\right): n=0,1, \ldots\right\}$ is homogeneous Markov chain with transition probabilities.

The function

$$
p_{i j}=P\left(X\left(\tau_{n+1}\right)=j \mid X\left(\tau_{n}\right)=i\right)=\lim _{t \rightarrow \infty} Q_{i j}(t) .
$$

$$
G_{i}(t)=P\left(T_{i} \leq t\right)=P\left(\tau_{n+1}-\tau_{n} \leq t \mid X\left(\tau_{n}\right)=i\right)=\sum_{j \in S} Q_{i j}(t)
$$

is the CDF of the random variable $T_{i}$ denoting time spent in state $i$ when the successor state is unknown. The function

$$
F_{i j}(t)=P\left(\tau_{n+1}-\tau_{n} \leq t \mid X\left(\tau_{n}\right)=i, X\left(\tau_{n+1}\right)=j\right)=\frac{Q_{i j}(t)}{p_{i j}}
$$

is CDF of a random variable $T_{i j}$ that is called a holding time of state $i$, if the next state will be $j$. From (6) we have

$$
Q_{i j}(t)=p_{i j} F_{i j}(t)
$$

The kernel of the SM process $\{X(t): t \geq 0\}$ takes the form

$$
Q(t)=\left[\begin{array}{llll}
0 & 0 & 0 & Q_{03}(t) \\
0 & 0 & 0 & Q_{13}(t) \\
Q_{20}(t) & Q_{21}(t) & 0 & Q_{23}(t) \\
Q_{30}(t) & 0 & Q_{32}(t) & 0
\end{array}\right]
$$

From (3) and from the assumptions we can calculate all elements of the semiMarkov kernel $Q(t), \quad t \geq 0$. The elements $Q_{03}(t)$ and $Q_{13}(t)$ are CDF of the system renewal time.

$$
Q_{03}(t)=Q_{13}(t)=H(t)=\int_{0}^{t} h(x) d x .
$$


From the system description and assumptions we get the following equalities:

$$
\begin{gathered}
Q_{20}(t)=P\left(U=0, \gamma_{A} \leq t, \zeta_{B}>\gamma_{A}\right)=(1-a) \int_{0}^{t} h_{A}(x)\left[1-F_{B}(x)\right] d x, \\
Q_{21}(t)=P\left(\zeta_{B} \leq t, \zeta_{B}<\gamma_{A}\right)=\int_{0}^{t} f_{B}(x)\left[1-H_{A}(x)\right] d x, \\
Q_{23}(t)=P\left(U=1, \gamma_{A} \leq t, \zeta_{B}>\gamma_{A}\right)=a \int_{0}^{t} h_{A}(x)\left[1-F_{B}(x)\right] d x .
\end{gathered}
$$

The similar way we obtain

$$
\begin{array}{r}
Q_{30}(t)=P\left(U=0, \zeta_{A} t\right)=(1-a) F_{A}(t), \\
Q_{32}(t)=P\left(U=1, \zeta_{A} t\right)=a F_{A}(t) .
\end{array}
$$

All elements of $Q(t)$ have been defined, hence the semi-Markov model describing the hospital power system evolution in reliability sense is constructed. It is necessary to calculate the transition probabilities of the embedded Markov chain. The transition probabilities matrix of the embedded Markov chain $\left\{X\left(\tau_{n}\right): n=0,1, \ldots\right\}$ is

$$
P=\left[\begin{array}{llll}
0 & 0 & 0 & 1 \\
0 & 0 & 0 & 1 \\
p_{20} & p_{21} & 0 & p_{23} \\
1-a & 0 & a & 0
\end{array}\right],
$$

where

$$
\begin{gathered}
p_{20}=(1-a) \int_{0}^{\infty} h_{A}(x)\left[1-F_{B}(x)\right] d x, \quad p_{21}=\int_{0}^{\infty} f_{B}(x)\left[1-H_{A}(x)\right] d x, \\
p_{23}=a \int_{0}^{\infty} h_{A}(x)\left[1-F_{B}(x)\right] d x .
\end{gathered}
$$

For supposing distributions for $t \geq 0$ we get

$$
\begin{gathered}
Q_{03}(t)=Q_{13}(t)=H(t)=1-(1+\mu t) e^{-\mu t} \\
Q_{20}(t)=\frac{(1-a) \mu_{A}^{2}}{\left(\alpha_{B}+\mu_{A}\right)^{2}}\left[1-\left(1+\left(\alpha_{B}+\mu_{A}\right) t\right) e^{-\left(\alpha_{B}+\mu_{A}\right) t}\right], \\
Q_{21}(t)=\frac{\alpha_{B}}{\left(\alpha_{B}+\mu_{A}\right)^{2}}\left[\alpha_{A}+2 \mu_{B}-\left(\alpha_{B}+\alpha_{B} \mu_{A} t+\mu_{A}\left(2+\mu_{A} t\right)\right) e^{-\left(\alpha_{B}+\mu_{A}\right) t}\right], \\
Q_{23}(t)=\frac{a \mu_{A}^{2}}{\left(\alpha_{B}+\mu_{A}\right)^{2}}\left[1-\left(1+\left(\alpha_{B}+\mu_{A}\right) t\right) e^{-\left(\alpha_{B}+\mu_{A}\right) t}\right],
\end{gathered}
$$


Reliability electrical power system of hospital as cold standby system

Niezawodność sytemu elektroenergetycznego szpitala jako niezawodność systemu...

$$
\begin{gathered}
Q_{30}(t)=(1-a)\left(1-e^{-\alpha_{A} t}\right), \\
Q_{32}(t)=a\left(1-e^{-\alpha_{A} t}\right) .
\end{gathered}
$$

The Laplace-Stielties transform of these functions are:

$$
\begin{gathered}
\tilde{q}_{03}(s)=\frac{\mu^{2}}{(s+\mu)^{2}}, \quad \tilde{q}_{13}(s)=\frac{\mu^{2}}{(s+\mu)^{2}} \\
\tilde{q}_{20}(s)=\frac{(1-a) \mu_{A}^{2}}{\left(s+\alpha_{B}+\mu_{A}\right)^{2}}, \quad \tilde{q}_{21}(s)=\frac{\alpha_{B}\left(s+\alpha_{B}+2 \mu_{A}\right)}{\left(s+\alpha_{B}+\mu_{A}\right)^{2}}, \\
\tilde{q}_{23}(s)=\frac{a \mu_{A}^{2}}{\left(s+\alpha_{B}+\mu_{A}\right)^{2}} \\
\tilde{q}_{30}(s)=\frac{(1-a) \alpha_{A}}{s+\alpha_{A}}, \quad \tilde{q}_{32}(s)=\frac{a \alpha_{A}}{s+\alpha_{A}} .
\end{gathered}
$$

The CDF of the waiting times are

$$
\begin{gathered}
G_{0}(t)=G_{1}(t)=Q_{03}(t)=Q_{13}(t)=1-(1+\mu t) e^{-\mu t}, \\
G_{2}(t)=Q_{20}(t)+Q_{21}(t)+Q_{23}(t)=1-\left(1+\mu_{A} t\right) e^{-\left(\alpha_{B}+\mu_{A}\right) t}, \\
G_{3}(t)=1-e^{-\alpha_{A} t} .
\end{gathered}
$$

The expected values of waiting times are

$$
E\left(T_{0}\right)=\frac{2}{\mu}, \quad E\left(T_{1}\right)=\frac{2}{\mu}, \quad E\left(T_{2}\right)=\frac{\alpha_{B}+2 \mu_{A}}{\left(\alpha_{B}+\mu_{A}\right)^{2}}, \quad E\left(T_{3}\right)=\frac{1}{\alpha_{A}} .
$$

\section{Reliability characteristic}

The function

$$
\Phi_{i D}(t)=P\left(\Theta_{D} \leq t \mid X(0)=i\right), t \geq 0
$$

is the CDF of a random variable $\Theta_{i D}$ denoting the first passage time from the state $i \in D^{\prime}$ to the subset $D$ or the exit time of $\{X(t): t 0\}$ from the subset $D^{\prime}$ with an initial state $i$. From the Theorem 1 of Chapter 4 [3] it follows that L-S transforms of the unknown CDF of the random variables $\Theta_{i D}, i \in D^{\prime}$ satisfy the system of linear equations (4.18) [3] which is equivalent to the matrix equation

$$
\left(I-q_{D^{\prime}}(s)\right) \varphi_{D^{\prime}}(s)=b(s),
$$

where $\quad I=\left[\delta_{i j}: i, j \in D^{\prime}\right] \quad$ is the unit matrix, $q_{D^{\prime}}(s)=\left[\tilde{q}_{i j}(s): i, j \in D^{\prime}\right]$ is the square sub-matrix of the matrix $q(s)$, while $\varphi_{D^{\prime}}(s)=\left[\tilde{\phi}_{i D}(s): i \in D^{\prime}\right]^{T}, \quad b(s)=\left[\sum_{j \in D} \tilde{q}_{i j}(s): i \in A^{\prime}\right]^{T} \quad$ are one column matrices of the corresponding L-S transforms. 
From the Theorem 2 of Chapter 3 [6] it follows that there exist expectations $E\left(\Theta_{i D}\right), \quad i \in D^{\prime}$ and they are unique solutions of the linear systems of equations, that have following matrix form

where

$$
\left(I-P_{D^{\prime}}\right) \bar{\Theta}_{D^{\prime}}=\bar{T}_{D^{\prime}}
$$

$$
P_{A^{\prime}}=\left[p_{i j}: i, j \in D^{\prime}\right], \quad \bar{\Theta}_{D^{\prime}}=\left[E\left(\Theta_{i D}\right): i \in D^{\prime}\right]^{T}, \quad \bar{T}_{D^{\prime}}=\left[E\left(T_{i}\right): i \in D^{\prime}\right]
$$

and $I$ is the unit matrix.

A main goal of this section is an evaluation a reliability function and a mean time to failure of the hospital power system. To get the reliability function we have to solve the matrix equation (22). In this case a set of "down" states is $D=\{0,1\}$ and the set of "up" states is $\{2,3\}$. The equation takes the form of

$$
\left[\begin{array}{ll}
1 & -\tilde{q}_{23}(s) \\
-\tilde{q}_{32}(s) & 1
\end{array}\right]\left[\begin{array}{l}
\tilde{\varphi}_{2 D}(s) \\
\tilde{\varphi}_{3 D}(s)
\end{array}\right]=\left[\begin{array}{l}
\tilde{q}_{20}(s)+\tilde{q}_{21}(s) \\
\tilde{q}_{30}(s)
\end{array}\right] .
$$

The solution of the equivalent linear equations system is

$$
\begin{gathered}
\tilde{\varphi}_{2 D}(s)=\frac{\tilde{q}_{20}(s)+\tilde{q}_{21}(s)+\tilde{q}_{23}(s) \tilde{q}_{30}(s)}{1-\tilde{q}_{23}(s) \tilde{q}_{32}(s)}, \\
\tilde{\varphi}_{3 D}(s)=\frac{\tilde{q}_{30}(s)+\tilde{q}_{32}(s) \tilde{q}_{20}(s)+\tilde{q}_{32}(s) \tilde{q}_{21}(s)}{1-\tilde{q}_{23}(s) \tilde{q}_{32}(s)} .
\end{gathered}
$$

Consequently, we obtain the Laplace transform of the reliability function

$$
\tilde{R}(s)=\frac{1-\widetilde{\varphi}_{3 D}(s)}{s} .
$$

The parameters of the model were estimated on the basis of few data and the opinion of the technical staff at one of the hospital in Poland. Therefore values of these parameters should be treated as approximate ones. The values of the model parameters are

$$
\mu=5.4, \quad a=0.99, \quad \alpha_{A}=0.0054, \alpha_{B}=0.164, \mu_{A}=4.2, \mu_{B}=3.1 .
$$

For these parameters we obtain the L-S transform $\tilde{\varphi}_{3 D}(s)$. Using the formula (26) we get the Laplace transform of the reliability function $\tilde{R}(s)$. We get the reliability function $R(t)$ of the system using the procedure InverseLaplaceTransform $[r, s, t]$ in the MATHEMATICA program. 
Reliability electrical power system of hospital as cold standby system

Niezawodność sytemu elektroenergetycznego szpitala jako niezawodność systemu...

Finally we obtain

$$
\begin{aligned}
& R(t)=1.000156 e^{-0.000497 t} \\
& -2[0.000078 \cos (0.146252 t)+0.000825 \sin (0.146252 t)] e^{-4.36645 t} \approx \\
& \approx e^{-0.000497 t} .
\end{aligned}
$$

From the well known equalities

$$
E(T)=\int_{0}^{\infty} R(t) d t, \quad E\left(T^{2}\right)=2 \int_{0}^{\infty} t R(t) d t, \quad D\left(T_{2}\right)=\sqrt{E\left(T^{2}\right)-[E(T)]^{2}}
$$

we get the mean time to failure and standard deviation of the system

$$
E(T)=2013.77[\text { days }] \approx 5.5[\text { years }], \quad D\left(T_{2}\right)=2013.45[\text { days }] .
$$

\section{References}

[1] Barlow, R.E. \& Proschan, F.: Statistical Theory of Reliability and Life Testing. Probability Models. Holt Rinehart and Winston, Inc., New York. 1975.

[2] Brodi SM, Pogosian JA: Embedded stochastic processes in theory of queue. Naukova Dumka, Kiev, 1973 (in Russian).

[3] Ferguson, T. S: A Bayesian analysis of some nonparametric problems. The Annals of Statistics, 1973.Vol.1, No. 2, 209-230.

[4] Grabski. F.. Some Method of Bayesian Nonparametric Estimation in Reliability. Proceeding of the European Conference on Safety and Reliability ESREL'98. Safety and Reliability. A.Balakema, Roterdam, Brookfield1998, p. 641-646.

[5] Grabski, F.: Semi-markowskie modele niezawodności i eksploatacji. Badania Systemowe, tom30 ; IBS PAN, Warszawa, 2002.

[6] Grabski, F. : Semi-Markov Processes: Application in System Reliability and Maintenance. Elsevier, Amsterdam, Boston, Heidelberg, London, New York Oxford, Paris,San Diego, San Francisco, Sydney,Tokyo, 2014.

[7] Korolyuk VS, Turbin AF. Semi-Markov processes and their applications. Naukova Dumka, Kiev 1976, (in Russian).

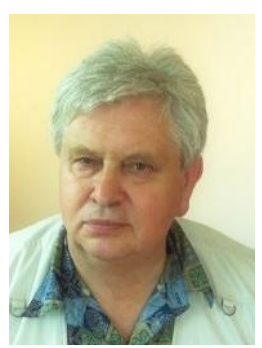

prof. dr hab. Franciszek Grabski Full Professor and Head of Department of Mathematics and Physics of Polish Naval Academy in Gdynia. Professor Grabski's main mathematical research interest focuses on probability theory, in particular its applications in system reliability theory and practice. He has constructed and tested several new reliability stochastic models and developed the Bayesian methods application in reliability. He is author or co-author more than 100 scientific papers, course-books and monographs in the probability and reliability field.(Udziat 100\%) 


\section{NIEZAWODNOŚĆ SYTEMU ELEKTROENERGETYCZNEGO SZPITALA JAKO NIEZAWODNOŚĆ SYSTEMU Z REZERWĄ ZIMNĄ}

\section{Wprowadzenie}

W pracy został przedstawiony stochastyczny model niezawodności systemu elektroenergetycznego, który składa się z systemu zasilania sieciowego, systemu zasilania awaryjnego i systemu przełączającego. Zasilanie sieciowe szpitala może zostać utracone w wyniku przerwania linii elektrycznych podczas prac ziemnych lub w przypadku linii napowietrznej w wyniku silnego wiatru, silnego zalodzenia, umyślnej dewastacji (kradzież) lub ataku terrorystycznego. Zanik napięcia sieciowego może trwać od kilku sekund do kilku godzin lub w skrajnych przypadkach kilku dni. Skutki takiej sytuacji mogą być różne dla różnych grup urządzeń. Awaria sieci powodująca natychmiastowe wyłączania urządzeń generuje duże ryzyko uszkodzenia sprzętu. Dotyczy to sprzętu ratowniczego, aparatury anestezjologicznej, dializatorów, ultrasonografów, tomografów itp. W przypadku wielu urządzeń medycznych jakakolwiek utrata zasilania powoduje zakłócenie ich pracy a po przywróceniu zasilania rozpoczynają się procesy autotestowania i samo-kalibracji, a urządzenia nie wykonują w tym czasie swoich podstawowych funkcji. W przypadku innych urządzeń, takich jak windy, lodówki, wentylatory przywrócenie zasilania nie powoduje tego typu perturbacji. W szpitalach większość systemów zasilania awaryjnego jest oparta na agregatach prądotwórczych napędzanych najczęściej silnikami benzynowymi, silnikami Disla lub silnikami gazowymi. Generator połączony jest zazwyczaj z automatycznym system wykrywania braku zasilania sieciowego i uruchomiania zasilania awaryjnego, który umownie nazywamy przełącznikiem. Osobną kwestią jest czas przełączania. Zwykle waha się od kilku do kilkudziesięciu sekund. Zazwyczaj generator uruchamia się automatycznie.

\section{Założenia modelu}

Żeby wyznaczyć charakterystyki niezawodnościowe systemu elektroenergetycznego szpitala skonstruujemy stochastyczny model funkcjonowania tego systemu w aspekcie niezawodności. Tym modelem będzie tak zwany włożony proces semimarkowski w proces opisujący działanie systemu [2]. Przedstawiony tu model jest modyfikacją opisu systemu $\mathrm{z}$ rezerwą zimną złożonego $\mathrm{z}$ dwóch różnych elementów $A$ i $B$ oraz przełącznika [5], [6]. Dodajmy, że systemy z rezerwą zimną złożone $\mathrm{z}$ dwóch identycznych elementów były rozpatrywane w pracach [1] oraz [7]. Element $A$ oznacza system zasilania sieciowego, element $B$ oznacza system zasilania awaryjnego natomiast $C$ oznacza system przełączający. Czas zdatności systemu zasilania sieciowego jest zmienną losową $\zeta_{A}$. 
Reliability electrical power system of hospital as cold standby system

Niezawodność sytemu elektroenergetycznego szpitala jako niezawodność systemu...

Charakter przyczyn uszkodzeń sieci elektrycznej pozwala przyjąć założenie, że ta zmienna losowa ma rozkład wykładniczy o gęstości $\mathrm{f}(x)=\propto_{A} e^{-\alpha_{A} x}, x \geq 0$. $\mathrm{W}$ chwili awarii systemu sieciowego $A$, natychmiast przełącznik uruchamia system awaryjny $B$, (czas przełączenia jest pomijany). Przyjmujemy, że czas naprawy systemu sieciowego $\gamma_{A}$ jest zmienną losową o rozkładzie określonym przez gęstość $h_{A}(x)=\mu_{A}^{2} x e^{-\mu_{A} x}, x \geq 0$. W chwili przywrócenia zdatności systemu zasilania sieciowego następuje przełączenie z zasilania awaryjnego na sieciowe. System awaryjny $B$ może również ulec uszkodzeniu a jego czas zdatności $\zeta_{B}$ jest zmienną losową o rozkładzie wykładniczym z parametrem $\alpha_{B}$. Totalna awaria sytemu elektroenergetycznego szpitala następuje wtedy, gdy awaryjny system zasilania $B$ ulegnie uszkodzeniu w czasie naprawy systemu podstawowego $A$ albo wtedy gdy nastąpi awaria systemu sieciowego i system przełączający okaże się niezdatny. Niech $U$ oznacza zmienną losową o rozkładzie binarnym $b(k)=P(U=k)=a^{k}(1-a)^{1-k}, k=0,1,0<a<1$, gdzie $U=0$, gdy przełącznik nie będzie działał $\mathrm{w}$ chwili uszkodzenia systemu zasilania sieciowego oraz $U=1 \mathrm{w}$ przeciwnym przypadku. Przyjmujemy, że czas przywrócenia pełnej zdatności całego systemu elektroenergetycznego jest zmienną losową $\gamma$ o gęstości $h(x), x \geq 0$. Ponadto zakładamy, że wszystkie tu rozpatrywane zmienne losowe są wzajemnie niezależne.

Głównym celem tej pracy jest oszacowanie funkcji niezawodności oraz wartości oczekiwanej czasu zdatności szpitalnego systemu elektroenergetycznego. Problem został rozwiązany przez zastosowanie 4-stanowego semi-markowskiego modelu niezawodności. Parametry modelu zostały oszacowane na podstawie niewielkiej liczby danych i opinii personelu technicznego w jednym ze szpitali w Polsce. Dlatego wartości tych parametrów powinny być traktowane jako przybliżone.

\section{Konstruowanie modelu}

Budowanie stochastycznego modelu niezawodności systemu elektroenergetycznego szpitala rozpoczynamy od określenia stanów:

0 - awaria całego systemu elektroenergetycznego spowodowana awarią podsystemu przełączającego $\mathrm{C}$,

1 - awaria całego systemu elektroenergetycznego spowodowana uszkodzeniem podsystemu awaryjnego $B \mathrm{w}$ czasie naprawy podsystemu zasilania sieciowego A,

2 - awaria podsystemu zasilania sieciowego $A$, (podsystem rezerwowy $B$ działa),

3 - zarówno podsystem zasilania sieciowego $A$, jak i podsystem rezerwowy $B$ są zdatne.

Przyjmujemy, że stanem początkowym jest 3 . Proces stochastyczny stanowiący model funkcjonowania systemu budujemy $\mathrm{w}$ następujący sposób: Niech $0=\tau_{0}^{*}$, $\tau_{1}^{*}, \tau_{2}^{*}, \ldots$ oznaczają chwile w których następują zmiany stanu systemu. 
Niech $\{Y(t): t \geq 0\}$ będzie procesem stochastycznym o zbiorze stanów $S=\{0,1,2,3\}$, prawostronnie ciągłych realizacjach, przyjmującym stałe wartości w przedziałach $\left[\tau_{n}^{*}, \tau_{n+1}^{*}\right), n=0,1, \ldots$. Ten proces na ogół nie jest procesem semi-Markowa (SM), gdyż własność braku pamięci nie jest zachowana we wszystkich chwilach zmian stanu. Można jednak skonstruować tak zwany włożony proces stochastyczny w proces $\{Y(t): t \geq 0\}$ [2], który będzie już procesem SM. Niech $0=\tau_{0}$ and $\tau_{1}, \tau_{2}, \ldots$ będą chwilami awarii podsystemów lub chwilami przywrócenia zdatności po awarii całego systemu. Proces stochastyczny $\{X(t): t \geq 0\}$ zdefiniowany wzorami

$$
X(0)=3, \quad X(t)=Y\left(\tau_{n}\right) \quad \text { dla } \quad t \in\left[\tau_{n}, \tau_{n+1}\right)
$$

jest już procesem semi-Markowa. Jest to proces wtożony $w$ proces stochastyczny $\{Y(t): t \geq 0\}$.

Żeby określić proces semi-Markowa musimy zdefiniować jego rozkład początkowy i wszystkie element jądra procesu [5], [6], [7]. Przypomnijmy, że jądro procesu jest macierzą funkcyjną

$$
\begin{aligned}
Q(t) & =\left[Q_{i j}(t): i, j \in S\right], \\
Q_{i j}(t)=P\left(\tau_{n+1}-\tau_{n}\right. & \left.\leq t, X\left(\tau_{n+1}\right)=j \mid X\left(\tau_{n}\right)=i\right), \quad t \geq 0 .
\end{aligned}
$$

Ciąg $\left\{X\left(\tau_{n}\right): n=0,1, \ldots\right\}$ jest jednorodnym lańcuchem Markowa o prawdopodobieństwach przejścia

Funkcja

$$
p_{i j}=P\left(X\left(\tau_{n+1}\right)=j \mid X\left(\tau_{n}\right)=i\right)=\lim _{t \rightarrow \infty} Q_{i j}(t),
$$

$$
G_{i}(t)=P\left(T_{i} \leq t\right)=P\left(\tau_{n+1}-\tau_{n} \leq t \mid X\left(\tau_{n}\right)=i\right)=\sum_{j \in S} Q_{i j}(t)
$$

jest dystrybuantą rozkładu zmiennej losowej $T_{i}$ oznaczającej czas trwania stanu $i$ bez względu na następny stan. Funkcja

$$
F_{i j}(t)=P\left(\tau_{n+1}-\tau_{n} \leq t \mid X\left(\tau_{n}\right)=i, X\left(\tau_{n+1}\right)=j\right)=\frac{Q_{i j}(t)}{p_{i j}}
$$

jest dystrybuantą rozkładu zmiennej losowej $T_{i j}$ oznaczającej czas trwania stanu $i$ gdy następnym stanem będzie $j$. Z (6) wynika równość

$$
Q_{i j}(t)=p_{i j} F_{i j}(t)
$$

Jądro procesu SM $\{X(t): t \geq 0\}$ ma postać

$$
Q(t)=\left[\begin{array}{llll}
0 & 0 & 0 & Q_{03}(t) \\
0 & 0 & 0 & Q_{13}(t) \\
Q_{20}(t) & Q_{21}(t) & 0 & Q_{23}(t) \\
Q_{30}(t) & 0 & Q_{32}(t) & 0
\end{array}\right]
$$


Reliability electrical power system of hospital as cold standby system

Niezawodność sytemu elektroenergetycznego szpitala jako niezawodność systemu...

Korzystając z (3) oraz założeń obliczamy wszystkie elementy jądra w $Q(t), t \geq 0$. Elementy $Q_{03}(t)$ oraz $Q_{13}(t)$ są dystrybuantami czasu odnowy.

$$
Q_{03}(t)=Q_{13}(t)=H(t)=\int_{0}^{t} h(x) d x .
$$

Z przytoczonych wyżej definicji i założeń otrzymujemy:

$$
\begin{gathered}
Q_{20}(t)=P\left(U=0, \gamma_{A} \leq t, \zeta_{B}>\gamma_{A}\right)=(1-a) \int_{0}^{t} h_{A}(x)\left[1-F_{B}(x)\right] d x, \\
Q_{21}(t)=P\left(\zeta_{B} \leq t, \zeta_{B}<\gamma_{A}\right)=\int_{0}^{t} f_{B}(x)\left[1-H_{A}(x)\right] d x \\
Q_{23}(t)=P\left(U=1, \gamma_{A} \leq t, \zeta_{B}>\gamma_{A}\right)=a \int_{0}^{t} h_{A}(x)\left[1-F_{B}(x)\right] d x
\end{gathered}
$$

Podobnie

$$
\begin{gathered}
Q_{30}(t)=P\left(U=0, \zeta_{A} \leq t\right)=(1-a) F_{A}(t) \\
Q_{32}(t)=P\left(U=1, \zeta_{A} \leq t\right)=a F_{A}(t)
\end{gathered}
$$

Wszystkie niezerowe element macierzy $Q(t)$ zostały zdefiniowane a więc niezawodnościowy stochastyczny model opisujący funkcjonowanie systemu elektroenergetycznego szpitala został zbudowany. Znajdziemy teraz macierz prawdopodobieństw przejścia włożonego łańcucha Markowa $\left\{X\left(\tau_{n}\right): n=0,1, \ldots\right\}$.

$$
P=\left[\begin{array}{llll}
0 & 0 & 0 & 1 \\
0 & 0 & 0 & 1 \\
p_{20} & p_{21} & 0 & p_{23} \\
1-a & 0 & a & 0
\end{array}\right]
$$

Korzystając ze wzorów (4), (8) oraz (9) - (14) otrzymujemy

$$
\begin{gathered}
p_{20}=(1-a) \int_{0}^{\infty} h_{A}(x)\left[1-F_{B}(x)\right] d x, \quad p_{21}=\int_{0}^{\infty} f_{B}(x)\left[1-H_{A}(x)\right] d x, \\
p_{23}=a \int_{0}^{\infty} h_{A}(x)\left[1-F_{B}(x)\right] d x .
\end{gathered}
$$

Dla przyjętych w założeniach rozkładów dla $t \geq 0$ otrzymujemy:

$$
\begin{gathered}
Q_{03}(t)=Q_{13}(t)=H(t)=1-(1+\mu t) e^{-\mu t}, \\
Q_{20}(t)=\frac{(1-a) \mu_{A}^{2}}{\left(\alpha_{B}+\mu_{A}\right)^{2}}\left[1-\left(1+\left(\alpha_{B}+\mu_{A}\right) t\right) e^{-\left(\alpha_{B}+\mu_{A}\right) t}\right],
\end{gathered}
$$




$$
\begin{gathered}
Q_{21}(t)=\frac{\alpha_{B}}{\left(\alpha_{B}+\mu_{A}\right)^{2}}\left[\alpha_{A}+2 \mu_{B}-\left(\alpha_{B}+\alpha_{B} \mu_{A} t+\mu_{A}\left(2+\mu_{A} t\right)\right) e^{-\left(\alpha_{B}+\mu_{A}\right) t}\right] \\
Q_{23}(t)=\frac{a \mu_{A}^{2}}{\left(\alpha_{B}+\mu_{A}\right)^{2}}\left[1-\left(1+\left(\alpha_{B}+\mu_{A}\right) t\right) e^{-\left(\alpha_{B}+\mu_{A}\right) t}\right] \\
Q_{30}(t)=(1-a)\left(1-e^{-\alpha_{A} t}\right), \\
Q_{32}(t)=a\left(1-e^{-\alpha_{A} t}\right) .
\end{gathered}
$$

Transformaty Laplace-Stieltiesa tych funkcji mają postać:

$$
\begin{gathered}
\tilde{q}_{03}(s)=\frac{\mu^{2}}{(s+\mu)^{2}}, \quad \tilde{q}_{13}(s)=\frac{\mu^{2}}{(s+\mu)^{2}} \\
\tilde{q}_{20}(s)=\frac{(1-a) \mu_{A}^{2}}{\left(s+\alpha_{B}+\mu_{A}\right)^{2}}, \quad \tilde{q}_{21}(s)=\frac{\alpha_{B}\left(s+\alpha_{B}+2 \mu_{A}\right)}{\left(s+\alpha_{B}+\mu_{A}\right)^{2}}, \quad \tilde{q}_{23}(s) \\
=\frac{a \mu_{A}^{2}}{\left(s+\alpha_{B}+\mu_{A}\right)^{2}}, \\
\tilde{q}_{30}(s)=\frac{(1-a) \alpha_{A}}{s+\alpha_{A}}, \quad \tilde{q}_{32}(s)=\frac{a \alpha_{A}}{s+\alpha_{A}} .
\end{gathered}
$$

Dystrybuanty rozkładów czasów trwania stanów $T_{\mathrm{i}}, 0,1,2,3$ wyrażają się wzorami:

$$
\begin{gathered}
G_{0}(t)=G_{1}(t)=Q_{03}(t)=Q_{13}(t)=1-(1+\mu t) e^{-\mu t} \\
G_{2}(t)=Q_{20}(t)+Q_{21}(t)+Q_{23}(t)=1-\left(1+\mu_{A} t\right) e^{-\left(\alpha_{B}+\mu_{A}\right) t} \\
G_{3}(t)=1-e^{-\alpha_{A} t}
\end{gathered}
$$

Wartości oczekiwane mają postać:

$$
E\left(T_{0}\right)=\frac{2}{\mu}, \quad E\left(T_{1}\right)=\frac{2}{\mu}, \quad E\left(T_{2}\right)=\frac{\alpha_{B}+2 \mu_{A}}{\left(\alpha_{B}+\mu_{A}\right)^{2}}, \quad E\left(T_{3}\right)=\frac{1}{\alpha_{A}} .
$$

\section{Charakterystyki niezawodności systemu}

Funkcja

$$
\Phi_{i D}(t)=P\left(\Theta_{D} \leq t \mid X(0)=i\right), t \geq 0
$$

jest dystrybuantą rozkładu zmiennej losowej $\Theta_{i D}$ oznaczającej czas pierwszego przejścia ze stanu $i \in D^{\prime}$ do podzbioru $D$ albo chwilę pierwszego opuszczenia przez proces of $\{X(t): t \geq 0\}$ podzbioru $D^{\prime}$ gdy stanem początkowym jest $i$. 
Reliability electrical power system of hospital as cold standby system

Niezawodność sytemu elektroenergetycznego szpitala jako niezawodność systemu...

$\mathrm{Z}$ twierdzenia 1 rozdziału $4 \mathrm{w}$ pracy [6] wynika, że transformaty LaplaceStieltiesa nieznanych dystrybuant zmiennych losowych $\Theta_{i D}, i \in D^{\prime}$ spełniają układ równań liniowych (4.18) [6], które w notacji macierzowej mają postać

$$
\left(I-q_{D^{\prime}}(s)\right) \varphi_{D^{\prime}}(s)=b(s),
$$

gdzie $I=\left[\delta_{i j}: i, j \in D^{\prime}\right]$ macierzą jednostkową, $q_{D^{\prime}}(s)=\left[\tilde{q}_{i j}(s): i, j \in D^{\prime}\right]$ jest podmacierzą macierzy $q(s)$, oraz $\varphi_{D^{\prime}}(s)=\left[\tilde{\phi}_{i D}(s): i \in D^{\prime}\right]^{T}, \quad b(s)=$ $\left[\sum_{j \in D} \tilde{q}_{i j}(s): i \in A^{\prime}\right]^{T} \quad$ są macierzami jednokolumnowymi odpowiednich transformat.

$\mathrm{Z}$ twierdzenia 1 rozdziału $4 \mathrm{w}$ pracy [6] wynika, że oczekiwany czas zdatności możemy otrzymać rozwiązując równanie macierzowe

gdzie

$$
\left(I-P_{D^{\prime}}\right) \bar{\Theta}_{D^{\prime}}=\bar{T}_{D^{\prime}}
$$

$$
P_{A^{\prime}}=\left[p_{i j}: i, j \in D^{\prime}\right], \bar{\Theta}_{D^{\prime}}=\left[E\left(\Theta_{i D}\right): i \in D^{\prime}\right]^{T}, \bar{T}_{D^{\prime}}=\left[E\left(T_{i}\right): i \in D^{\prime}\right] .
$$

W rozpatrywanym modelu podzbiór $D=\{0,1\}$ jest zbiorem stanów niezdatności natomiast $D^{\prime}=\{2,3\}$ jest podzbiorem zdatności systemu. Równanie

$$
\left[\begin{array}{ll}
1 & -\tilde{q}_{23}(s) \\
-\tilde{q}_{32}(s) & 1
\end{array}\right]\left[\begin{array}{l}
\tilde{\varphi}_{2 D}(s) \\
\tilde{\varphi}_{3 D}(s)
\end{array}\right]=\left[\begin{array}{l}
\tilde{q}_{20}(s)+\tilde{q}_{21}(s) \\
\tilde{q}_{30}(s)
\end{array}\right] .
$$

Rozwiązanie równania (22) w rozpatrywanym tu przypadku prowadzi do wzorów.

$$
\begin{gathered}
\tilde{\varphi}_{2 D}(s)=\frac{\tilde{q}_{20}(s)+\tilde{q}_{21}(s)+\tilde{q}_{23}(s) \tilde{q}_{30}(s)}{1-\tilde{q}_{23}(s) \tilde{q}_{32}(s)}, \\
\tilde{\varphi}_{3 D}(s)=\frac{\tilde{q}_{30}(s)+\tilde{q}_{32}(s) \tilde{q}_{20}(s)+\tilde{q}_{32}(s) \tilde{q}_{21}(s)}{1-\tilde{q}_{23}(s) \tilde{q}_{32}(s)} .
\end{gathered}
$$

Transformatę Laplace'a funkcji niezawodności rozpatrywanego systemu obliczamy korzystając ze związku

$$
\tilde{R}(s)=\frac{1-\widetilde{\varphi}_{3 D}(s)}{s} .
$$

Parametry modelu zostały oszacowane metodą Fergusona [3], [4] w oparciu o opinie ekspertów (pracowników technicznych szpitala) i nieliczne dane statystyczne dotyczące jednego ze szpitali w Polsce. Oszacowane wartości parametrów są następujące:

$\mu=5.4, \quad a=0.99, \quad \alpha_{A}=0.0054, \alpha_{B}=0.164, \mu_{A}=4.2, \mu_{B}=3.1$.

Dla tych parametrów podstawiając do wzorów (25) transformaty (18), otrzymujemy transformatę $\tilde{\varphi}_{3 D}(s)$. Korzystając ze wzoru (26) otrzymujemy transformatę Laplace'a $\tilde{R}(s)$ funkcji niezawodności sytemu. Chcąc znaleźć funkcję niezawodności $R(t)$ jako transformatę odwrotną skorzystaliśmy $\mathrm{z}$ 
procedury InverseLaplaceTransform $[r, s, t]$ w programie MATHEMATICA. Ostatecznie otrzymujemy:

$R(t)=1.000156 e^{-0.000497 t}$

$-2[0.000078 \cos (0.146252 t)+0.000825 \sin (0.146252 t)] e^{-4.36645 t} \approx$

$\approx \exp [-0.000497 \mathrm{t}]$

Korzystając z dobrze znanych wzorów

$$
E(T)=\int_{0}^{\infty} R(t) d t, \quad E\left(T^{2}\right)=2 \int_{0}^{\infty} t R(t) d t, \quad D\left(T_{2}\right)=\sqrt{E\left(T^{2}\right)-[E(T)]^{2}}
$$

otrzymujemy wartość oczekiwaną i odchylenie standardowe czasu zdatności systemu elektroenergetycznego szpitala:

$$
E(T)=2013.77[d n i] \approx 5.5[\text { lat }], \quad D\left(T_{2}\right)=2013.45[d n i] .
$$

\section{Literatura}

[1] Barlow, R.E. \& Proschan, F.: Statistical Theory of Reliability and Life Testing. Probability Models. Holt Rinehart and Winston, Inc., New York. 1975.

[2] Brodi SM, Pogosian JA: Embedded stochastic processes in theory of queue. Naukova Dumka, Kiev, 1973 (in Russian).

[3] Ferguson, T. S: A Bayesian analysis of some nonparametric problems. The Annals of Statistics, 1973.Vol.1, No. 2, 209-230.

[4] Grabski. F.. Some Method of Bayesian Nonparametric Estimation in Reliability. Proceeding of the European Conference on Safety and Reliability ESREL'98. Safety and Reliability. A.Balakema, Roterdam, Brookfield1998, p. 641-646.

[5] Grabski, F.: Semi-markowskie modele niezawodności i eksploatacji. Badania Systemowe, tom30; IBS PAN, Warszawa, 2002.

[6] Grabski, F. : Semi-Markov Processes: Application in System Reliability and Maintenance. Elsevier, Amsterdam, Boston, Heidelberg, London, New York Oxford, Paris,San Diego, San Francisco, Sydney,Tokyo, 2015.

[7] Korolyuk VS, Turbin AF. Semi-Markov processes and their applications. Naukova Dumka, Kiev 1976, (in Russian).

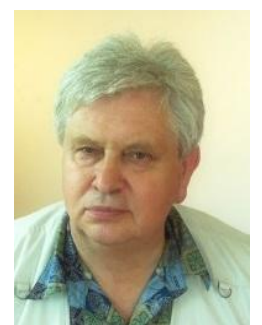

prof. dr hab. Franciszek Grabski Full Professor and Head of Department of Mathematics and Physics of Polish Naval Academy. Professor Grabski's main mathematical research interest focuses on probability theory, in particular its applications in system reliability theory and practice. He has constructed and tested several new reliability stochastic models and developed the Bayesian methods application in reliability. He is author or co-author more than 100 scientific papers, course-books and monographs in the probability and reliability field. (Share: 100\%). 
Reliability electrical power system of hospital as cold standby system Niezawodność sytemu elektroenergetycznego szpitala jako niezawodność systemu... 